\title{
Development of a 95 SNP Panel To Individually Genotype Mountain Lions (Puma Concolor) For Microfluidic and Other Genotyping Platforms
}

Michael Buchalski ( $\nabla$ michael.buchalski@wildlife.ca.gov )

California Department of Fish and Wildlife https://orcid.org/0000-0002-5917-3577

Benjamin Sacks

University of California Davis

Kristen Ahrens

CDFW: California Department of Fish and Wildlife

Kyle Gustafson

Arkansas State University

Jaime Rudd

CDFW: California Department of Fish and Wildlife

Holly Ernest

University of Wyoming

Justin Dellinger

CDFW: California Department of Fish and Wildlife

\section{Research Article}

Keywords: California, fecal DNA, Fluidigm, Mountain lion, Puma concolor, single nucleotide polymorphism

Posted Date: October 18th, 2021

DOI: https://doi.org/10.21203/rs.3.rs-891750/v1

License: (a) (1) This work is licensed under a Creative Commons Attribution 4.0 International License. Read Full License

Version of Record: A version of this preprint was published at Conservation Genetics Resources on February 23rd, 2022. See the published version at https://doi.org/10.1007/s12686-022-01255-6. 


\section{Abstract}

The mountain lion (Puma concolor) is one of the few remaining large predators in California, USA with density estimation from fecal genotypes becoming an essential component of conservation and management. In highly urbanized southern California, mountain lions are fragmented into small, inbred populations making proper marker selection critical for individual identification. We developed a panel of single nucleotide polymorphism (SNP) markers that can be used for consistent, routine mountain lion monitoring by different laboratories. We used a subset of existing Illumina HiSeq data for 104 individuals from throughout California to design a single, highly heterozygous multiplex of 95 SNPs for the Fluidigm platform. This panel confidently differentiates individual mountain lions, identifies sex, and discriminates mountain lions from bobcats. The panel performed well on fecal DNA extracts and based on design, had sufficient resolution to differentiate individual genotypes in even the population with lowest genetic diversity in southern California.

\section{Introduction}

The mountain lion (Puma concolor) is an elusive, common large predator native to California, USA. Monitoring of wild felids often requires non-invasive genetic tools (Ernest et al. 2000). In California, population monitoring has increasingly relied on canine surveys for feces followed by fecal genotyping and mark-recapture analyses to estimate population abundance. Although microsatellites have previously been relied upon for such noninvasive surveys, they have several weaknesses that must be overcome to prevent errors (Taberlet et al. 1999), including low amplification rates (Brinkman et al. 2010; Murphy et al. 2007; Piggott 2004), challenges calibrating between laboratories (von Thaden et al. 2017), allelic dropout, and false alleles (Creel et al. 2003; Pompanon et al. 2005).

There is great need for genetic methods that can be applied to low quality DNA samples while maintaining statistical power to differentiate individuals in low-diversity populations. In southern California, many mountain lion populations are impacted by urban development, and exist in small numbers with low genetic diversity (Ernest et al. 2014; Gustafson et al. 2017; Riley et al. 2014), making it difficult to apply genetic methods without high probability of monomorphic loci and identification errors. A previous SNP panel (PumaPlex100; Erwin et al. 2021) demonstrated the utility of SNPs to differentiate among mountain lion individuals. However, this panel was not optimized for low-diversity populations and requires up to four multiplexes. Therefore, we developed a single multiplex of 95 SNPs for the Fluidigm platform (Fluidigm, San Francisco USA) that can be used across laboratories for surveys of California and potentially other mountain lion populations. These SNPs can identify individuals for abundance monitoring, assign sex, and discriminate mountain lion feces from bobcat (Lynx rufus), the most common non-target species detected during canine surveys.

We identified SNPs using existing RADseq data (Illumina HiSeq, paired-end 150 bp reads) from mountain lion tissues sampled as part of a California statewide population genetic study (Gustafson et al. in prep.) and mapped reads to the Puma concolor scaffold-level genome assembly (PumCon1.0), using 104 
individuals, including up to 15 individuals from each of 10 populations identified by Gustafson et al. (2019). We used vcftools to filter biallelic autosomal SNPs to a PHRED score $\geq 30$ ( $99.9 \%$ accuracy) with $\geq 80 \%$ of individuals genotyped at each SNP. To maximize heterozygosity and resolution to differentiate individuals, we used plink v1.90b6.10 to filter the minor allele frequency to $0.40-0.45$. We retained 134 SNPs distributed across 28 scaffolds (mean within-scaffold distance of $11.3 \mathrm{Mbp}$ ) for further validation. We identified species-diagnostic SNPs using published mitogenome sequences for mountain lion and bobcat (Supporting information Table S1). We also identified sex-linked SNPs from both the SRY locus on the non-recombining region of the $Y$ chromosome and the zinc finger orthologs of the $X$ and $Y$ chromosomes (Supporting information, Table S2).

We used pysam v0.15.0 to retrieve 100 base pairs of up- and down-stream flanking sequence for the 134 SNPs. We used Fluidigm's D3 Assay Design Tool (https://d3.fluidigm.com) in conjunction with the flanking sequences (Supporting Information, Table S3) to design primers, which we ordered from Fluidigm (Supporting information, Table S4).

To test the performance of the SNP panel on fecal DNA, we used 19 pairs of tissue and fecal samples collected during mountain lion necropsies conducted by the California Department of Fish and Wildlife from 14 different counties. Feces were experimentally weathered outdoors in direct sunlight for seven days. Tissue and fecal DNA were extracted using the Genomic Tip 20G kit and the QIAamp Fast DNA Stool Mini Kit (Qiagen, Valencia, CA), respectively. To assess the performance of the SNP panel on lowdiversity populations, we extracted tissues for 26 mountain lions from the highly inbred Santa Ana Mountains population in southern California. We also extracted 16 bobcat tissues to test the speciesdiagnostic SNPs.

We used Fluidigm's protocol for 96.96 Dynamic Arrays with integrated fluidic circuits run on the Juno thermocycler paired with the Biomark HD system. We modified the pre-amplification PCR reaction to align with optimizations for degraded samples (see Table S2 in von Thaden et al. 2020). We also increased the manufacturer's PCR cycling profile from 34 to 45 in the final allele specific reaction. We analyzed genotyping results using Fluidigm SNP Genotyping Analysis Software version 4.5.1. We selected a final panel of 91 autosomal SNPs based on consistent cluster separation and high call rates. We also selected two species-diagnostic and two sex-linked SNPs. For the Santa Ana population, we estimated expected heterozygosity $H_{E}$, probability of identity $\mathrm{P}_{\mathrm{ID}}$, probability of identity in siblings $\mathrm{P}_{\mathrm{IDsibs}}$, and calculated the genotype accumulation curve using the adegenet, popgenutils, and poppr packages in $\mathrm{r}$ (Jombart and Ahmed 2011; Kamvar et al. 2014).

When genotyping the 19 paired tissue and fecal samples at the 91 autosomal SNPs, we obtained $>95 \%$ call rates on 18 of the 19 fecal samples. The overall agreement between the paired samples was $99.9 \%$ $(S D=0.6 \%)$, indicating a genotyping error rate $<0.1 \%$. For the low-diversity Santa Ana population, the average $H_{\mathrm{E}}$ for the 91 autosomal SNPs was 0.404 , an estimate higher than previously measured using 42 microsatellites ( $H_{\mathrm{E}}=0.33$; Gustafson et al. 2019), suggesting our SNP filtering protocol successfully inflated heterozygosity and increased power for individual identification. In fact, values for $P_{I D}$ and $P_{I D s i b s}$ 
across all SNPs were $<1 \times 10^{-10}$ (Supporting information, Table S5) and individual identifications could be made with high confidence from as few as 15 SNPs (Fig. 1). For 33 samples where both sex-linked SNPs (2FelidSRYSNP-GT, mlbcZfy-680) yielded genotypes, 30 pairs (91\%) agreed with known sex, suggesting < $5 \%$ sex-typing error rate. The species-diagnostic SNPs (mtdna_658, mtdna_2089) were 100\% successful at differentiating mountain lions from bobcats. Further, bobcat reference genotypes were monomorphic at all autosomal SNPs.

This high-resolution panel of 95 SNPs was optimized for the Fluidigm system but can be genotyped in any SNP-typing platform. The panel was designed from mountain lions throughout California and therefor is subject to ascertainment bias when applied to other geographic regions. The high minor allele frequency screening step for SNP selection proved useful for differentiating individuals but also inflates estimates of heterozygosity. The utility of this panel for individual identification throughout the species range requires further verification.

\section{Declarations}

\section{Acknowledgements}

Funding for this project was from the U.S. Fish and Wildlife Service, Wildlife Restoration Act and the California Department of Fish and Wildlife, Big Game Management Program (Grant No. P1580009). S. Vanderzwan and A. Kubicki were instrumental to completing the laboratory work

Funding was from the U.S. Fish and Wildlife Service, Wildlife Restoration Act and the California Department of Fish and Wildlife, Big Game Management Program (Grant No. P1580009)

Conflicts of interest/Competing interests: Not applicable

Availability of data and material: Provided upon request

Author contributions: Dellinger and Rudd collected the samples; Buchalski designed the study; Buchalski, Gustafson, Ernest, and Sacks discovered the SNPs; Sacks and Ahrens tested and selected the final panel; all authors contributed to writing.

\section{References}

1. Brinkman TJ, Schwartz MK, Person DK, Pilgrim KL, Hundertmark KJ (2010) Effects of time and rainfall on PCR success using DNA extracted from deer fecal pellets. 11(4): 1547-1552

2. Creel S, Spong G, Sands JL, Rotella J, Zeigle J, Joe L et al (2003) Population size estimation in Yellowstone wolves with error-prone noninvasive microsatellite genotypes. Mol Ecol 12(7):20032009

3. Ernest HB, Penedo MC, May BP, Syvanen M, Boyce WM (2000) Molecular tracking of mountain lions in the Yosemite valley region in California: genetic analysis using microsatellites and faecal DNA. 
Mol Ecol 9(4):433-441

4. Ernest HB, Vickers TW, Morrison SA, Buchalski MR, Boyce WM (2014) Fractured genetic connectivity threatens a southern California puma (Puma concolor) population. 9: e107985

5. Erwin JA, Fitak RR, Culver M (2021) PumaPlex100: an expanded tool for puma SNP genotyping with low-yield DNA

6. Gustafson KD, Gagne RB, Vickers TW, Riley SPD, Wilmers CC, Bleich VC et al (2019) Genetic sourcesink dynamics among naturally structured and anthropogenically fragmented puma populations. 20(2): 215-227

7. Gustafson KD, Vickers TW, Boyce WM, Ernest HB (2017) A single migrant enhances the genetic diversity of an inbred puma population. Royal Society open science 4(5):170115

8. Jombart T, Ahmed I (2011) adegenet 1.3-1: new tools for the analysis of genome-wide SNP data. Bioinformatics 27(21):3070-3071

9. Kamvar ZN, Tabima JF, Grünwald NJ (2014) Poppr: an R package for genetic analysis of populations with clonal, partially clonal, and/or sexual reproduction. PeerJ 2:e281

10. Murphy MA, Kendall KC, Robinson A, Waits LP (2007) The impact of time and field conditions on brown bear (Ursus arctos) faecal DNA amplification. 8(5): 1219-1224

11. Piggott M (2004) Effect of sample age and season of collection on the reliability of microsatellite genotyping of faecal DNA. 31(5): 485-493

12. Pompanon F, Bonin A, Bellemain E, Taberlet P (2005) Genotyping errors: causes, consequences and solutions. Nature reviews Genetics 6(11):847-859

13. Riley SP, Serieys LE, Pollinger JP, Sikich JA, Dalbeck L, Wayne RK et al (2014) Individual behaviors dominate the dynamics of an urban mountain lion population isolated by roads. Current biology: CB 24(17):1989-1994

14. Taberlet P, Waits LP, Luikart G (1999) Noninvasive genetic sampling: look before you leap. Trends Ecol Evol 14(8):323-327

15. von Thaden A, Cocchiararo B, Jarausch A, Jüngling H, Karamanlidis A, Tiesmeyer A et al (2017) Assessing SNP genotyping of noninvasively collected wildlife samples using microfluidic arrays. 7: 10768

16. von Thaden A, Nowak C, Tiesmeyer A, Reiners TE, Alves PC, Lyons LA et al (2020) Applying genomic data in wildlife monitoring: development guidelines for genotyping degraded samples with reduced single nucleotide polymorphism panels. 20(3): 662-680

\section{Figures}




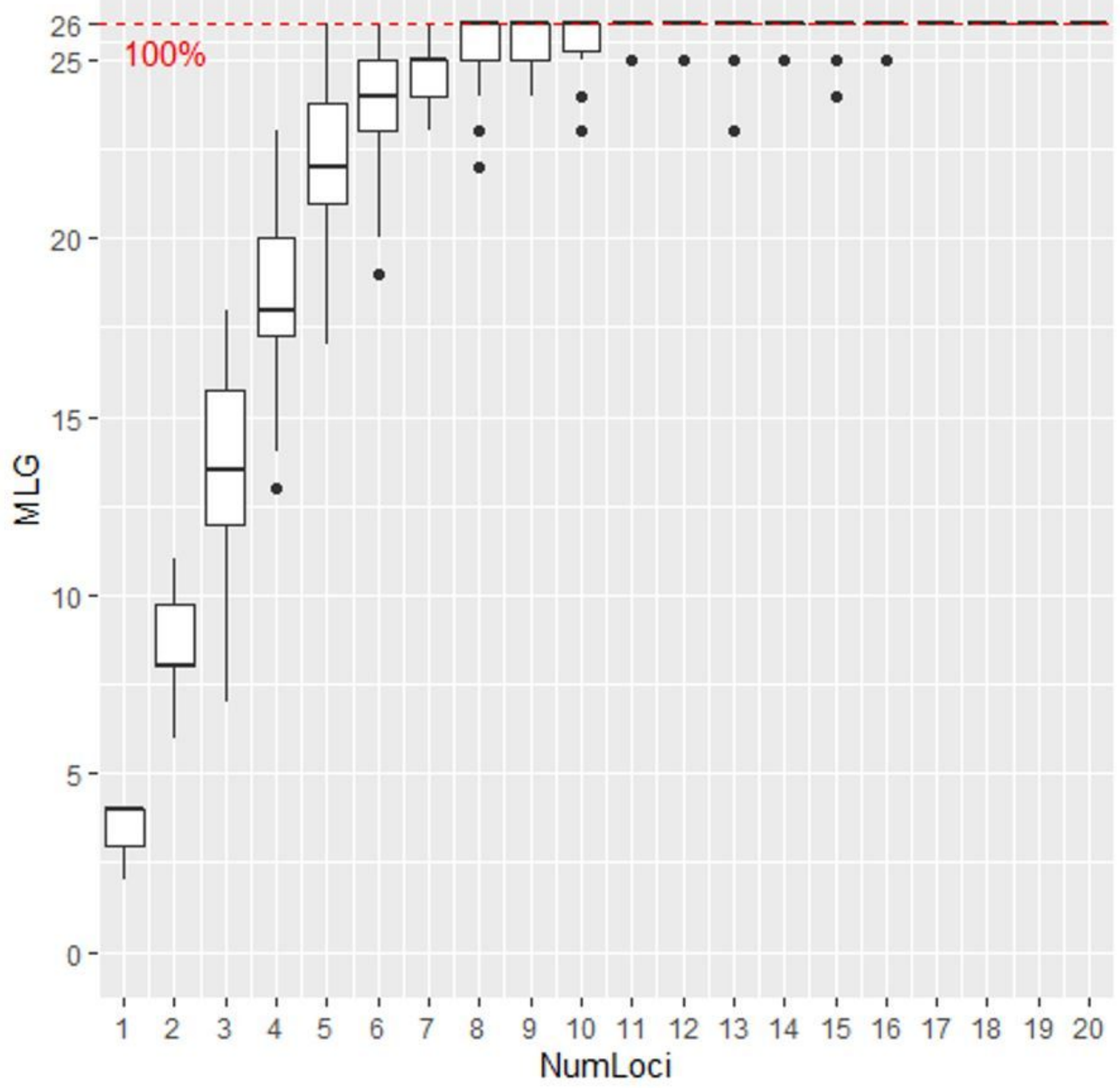

Figure 1

Genotype accumulation curve for 26 mountain lions from the low-diversity Santa Ana population. Estimated number of multilocus genotypes (MLG) identified based on the number of SNP loci sampled and 1,000 randomizations of the data

\section{Supplementary Files}

This is a list of supplementary files associated with this preprint. Click to download.

- TablesS3S5.xls 